\title{
LAPLACE-RUNGE-LENZ VECTOR IN QUANTUM MECHANICS IN NONCOMMUTATIVE SPACE
}

\author{
Peter Prešnajder*, Veronika Gáliková, Samuel Kováčik \\ Commenius University Bratislava, Faculty of Mathematics, Physics and Informatics \\ * corresponding author: presnajder.peter@gmail.com
}

\begin{abstract}
The object under scrutiny is the dynamical symmetry connected with conservation of the Laplace-Runge-Lenz vector (LRL) in the hydrogen atom problem solved by means of noncommutative quantum mechanics (NCQM). The considered noncommutative configuration space has such a "fuzzy" structure that the rotational invariance is not spoilt. An analogy with the LRL vector in the NCQM is brought to provide our results and also a comparison with the standard QM predictions.
\end{abstract}

KEYWORDS: noncommutative space, Coulomb-Kepler problem, symmetry.

\section{INTRODUCTION}

Our main goal we are after is to investigate the existence of dynamical symmetry of the Coulomb-Kepler problem in the quantum mechanics in noncommutative space, and possibly to find the generalization of the so-called Laplace-Runge-Lenz (LRL) vector for this case. Before actually starting, we briefly look at the history of the LRL vector. We call this Laplace-Runge-Lenz vector, as it is commonly named nowadays, but as far as we know, the first ones to make a mention of it were Jakob Hermann and Johann Bernoulli in the letters they exchanged in 1710, see [1, [2]. So the name "Hermann-Bernoulli vector" would be more proper. Much later, in 1799 the vector was rediscovered by Laplace in his Celestial Mechanics [3]. Then it appeared in a popular German textbook on vectors by $C$. Runge [4, which was referenced by $W$. Lenz in his paper on the (old) quantum mechanical treatment of the Kepler problem or hydrogen atom [5].

Now back to physics. The Coulomb-Kepler problem is all about the motion of a particle in a field of a central force proportional to $\left(r^{-2}\right)$. The corresponding Newton equation for a body of mass $m$ reads

$$
m \dot{\vec{v}}=-q \frac{\vec{r}}{r^{3}} .
$$

Here $q$ denotes a constant which specifies the magnitude of the force applied. The system with a central force definitely is rotationally symmetric and the orbital momentum,

$$
\vec{L}=m \vec{r} \times \vec{v},
$$

is conserved in any central field. However, as to the symmetries, more has to be said in this case, due to the fact that not only is the force central, but in addition it has the inverse square dependence of the distance. Besides the components of $\vec{L}$, the CoulombKepler problem has three additional integrals of motion, namely those represented by the conserved LRL vector,

$$
\vec{A}=\vec{L} \times \vec{v}+q \frac{\vec{r}}{r}
$$

When the motion of a planet around the Sun is considered, the conservation of the given quantity has to do with the constant eccentricity of the orbit and the position of the perihelion.

Another well-known system characterized by Coulomb potential is the hydrogen atom. Obviously a need for the use of quantum mechanics arises here. There are, however, several ways to address the issue. In 1926 Wolfgang Pauli published his paper on the subject [6]. He used the LRL vector to find the spectrum of a hydrogen atom using modern quantum mechanics and the hidden dynamical symmetry of the problem, without knowledge of the explicit solution of the Schrödinger equation. It turned out that the LRL vector can be found among the hermitian operators acting in the Hilbert space considered in an almost complete analogy with the classical case, the only subtlety to deal with being the fact that the cross product needs to be properly symmetrized, resulting in

$$
A_{k}^{Q M}=\frac{1}{2} \varepsilon_{i j k}\left(L_{i} v_{j}+v_{j} L_{i}\right)+q \frac{x_{k}}{r},
$$

where $v_{j}=-\frac{i \hbar}{m} \partial_{j}$ stands for velocity operator. Importantly the operators $L_{i}$ and $A_{i}$ commute with the Hamiltonian, i.e. are conserved with respect to time evolution, and as to their mutual commutation relations, there would be pretty good views of them forming a closed algebra $s o(4)$, if it were not for the commutator $\left[A_{i}, A_{j}\right] \propto L_{k} H$. However, restricting ourselves to the subspace $\mathcal{H}_{E}$ spanned by eigenvectors of $H$ corresponding to the eigenvalue $\mathrm{E}, H$ can be replaced by its eigenvalue, which is a c-number. Besides enabling the algebra to close, we have also dragged the energy into the very definition of the algebra generators. This, together with the theory related to the relevant Casimir operators, has a direct impact on the H-atom energy spectrum. In this way Pauli found the correct formulas for the hydrogen atom spectrum even before Schrödinger.

Now the search for the analogy in noncommutative quantum mechanics (NCQM) begins. The rest of this paper is organized in the following way: In Section 2 
we give a brief introduction to the quantum mechanics in a spherically symmetric noncommutative space. The Hilbert space of wave functions in NC space and the NC generalizations of important operators (Hamiltonian, angular momentum, coordinate and velocity) are introduced. When we use these, a generalization of the dynamical symmetry of the Coulomb-Kepler problem in NCQM is presented in Section 3. Section 4 provides conclusions. We skip all the detailed and lengthy calculations that can be found in our recently published paper [7].

\section{BASICS OF NONCOMMUTATIVE QUANTUM MECHANICS}

To begin, it should be made clear how to introduce some uncertainty principle into the configuration space for the Coulomb problem without spoiling the key feature which allows us to find the exact solution the rotational invariance.

The uncertainty is expressed as nontrivial commutation relations for the $\mathrm{NC}$ analogs of the former Cartesian coordinates (obviously we have to abandon c-numbers), defined in a spherically symmetrical way.

The coordinates in the NC configuration space $\mathbf{R}_{\lambda}^{3}$ are realized in terms of 2 pairs of boson annihilation and creation operators $a_{\alpha}, a_{\alpha}^{+}, \alpha=1,2$, satisfying

$$
\left[a_{\alpha}, a_{\beta}^{+}\right]=\delta_{\alpha \beta},\left[a_{\alpha}, a_{\beta}\right]=\left[a_{\alpha}^{+}, a_{\beta}^{+}\right]=0 .
$$

They act in an auxiliary Fock space $\mathcal{F}$ spanned by the normalized vectors

$$
\left|n_{1}, n_{2}\right\rangle=\frac{\left(a_{1}^{+}\right)^{n_{1}}\left(a_{2}^{+}\right)^{n_{2}}}{\sqrt{n_{1} ! n_{2} !}}|0\rangle .
$$

The normalized vacuum state is denoted as $|0\rangle \equiv$ $|0,0\rangle$.

The noncommutative coordinates $x_{j}, j=$ $1,2,3$, and the NC analog of the Euclidean distance from the origin are given as

$$
\begin{aligned}
x_{j} & =\lambda a^{+} \sigma^{j} a \equiv \lambda \sigma_{\alpha \beta}^{j} a_{\alpha}^{+} a_{\beta}, \\
r & =\lambda(N+1),
\end{aligned}
$$

where $\sigma^{j}$ are the Pauli matrices, $N=a_{\alpha}^{+} a_{\alpha}$ is the number operator in the Fock space, and $\lambda$ is a length parameter. Its magnitude is not fixed within our model. Naturally it has a connection with the smallest distance relevant in the given noncommutative configuration space denoted as $\mathbf{R}_{\lambda}^{3}$. The key rotationally invariant relations in the theory are

$$
\left[x_{i}, x_{j}\right]=2 i \lambda \varepsilon_{i j k} x_{k}, \quad\left[x_{i}, r\right]=0, \quad r^{2}-x_{j}^{2}=\lambda^{2} .
$$

The first equation defines a noncommutative or fuzzy sphere that appeared a long time ago in various contexts [8], e.g., quantization on a sphere $=$ nonflat phase space, a simple model of NC manifolds. All these models considered a single fuzzy sphere. Here we deal with an infinite sequence of fuzzy spheres dynamically via an NC analog of the (radial) Schrödinger equation that is introduced below. We remark that while the first equation in 8 is postulated, the other two follow from the construction of $\mathbf{R}_{\lambda}^{3}$.

While constructing $\mathrm{NC}$ quantum mechanics, firstly we have to decide on a Hilbert space $\mathcal{H}_{\lambda}$ of states (see also 9]). The suitable choice is a linear space of normally-ordered analytic functions containing the same number of creation and annihilation operators:

$$
\Psi=\sum C_{m_{1} m_{2} n_{1} n_{2}}\left(a_{1}^{+}\right)^{m_{1}}\left(a_{2}^{+}\right)^{m_{2}} a_{1}^{n_{1}} a_{2}^{n_{2}},
$$

which possess finite weighted Hilbert-Schmidt norm

$$
\|\Psi\|^{2}=4 \pi \lambda^{2} \operatorname{Tr}\left[r \Psi^{\dagger} \Psi\right] .
$$

(The summation in 9 is over nonnegative integers satisfying $m_{1}+m_{2}=n_{1}+n_{2}$.)

Our NC wave functions $\Psi$ are themselves operators on the Fock space mentioned above, so the relations which occur are to be looked at as operator equalities (we could put $\left|n_{1}, n_{2}\right\rangle$ on both sides of every such equation).

We can move to the definition of the operators acting on $\mathrm{NC}$ wave functions $\Psi \in \mathcal{H}_{\lambda}$. To avoid potential confusion, we have decided to leave the NC coordinates and the $\mathrm{NC}$ wave functions $\Psi$ (operators on the Fock space) as they are, and to denote the operators acting on $\Psi$ with a hat from now on.

The generators of rotations in $\mathcal{H}_{\lambda}$, orbital momentum operators, are defined as

$$
\hat{L}_{j} \Psi=\frac{1}{2 \lambda}\left(x_{j} \Psi-\Psi x_{j}\right), \quad j=1,2,3 .
$$

They are hermitian and obey the usual commutation relations

$$
\left[\hat{L}_{i}, \hat{L}_{j}\right] \Psi \equiv\left(\hat{L}_{i} \hat{L}_{j}-\hat{L}_{j} \hat{L}_{i}\right) \Psi=i \varepsilon_{i j k} \hat{L}_{k} \Psi .
$$

The standard eigenfunctions $\Psi_{j m}, j=0,1,2, \ldots$, $m=-j, \ldots,+j$, satisfying

$$
\hat{L}_{i}^{2} \Psi_{j m}=j(j+1) \Psi_{j m}, \hat{L}_{3} \Psi_{j m}=m \Psi_{j m},
$$

are given by

$$
\Psi_{j m}=\sum_{(j m)} \frac{\left(a_{1}^{+}\right)^{m_{1}}\left(a_{2}^{+}\right)^{m_{2}}}{m_{1} ! m_{2} !} R_{j}(r) \frac{a_{1}^{n_{1}}\left(-a_{2}\right)^{n_{2}}}{n_{1} ! n_{2} !} .
$$

The summation goes over all nonnegative integers that satisfy

$$
m_{1}+m_{2}=n_{1}+n_{2}=j, m_{1}-m_{2}-n_{1}+n_{2}=2 m .
$$

For any fixed $R_{j}(r)$ equation (14) defines a representation space for a unitary irreducible representation with spin $j$.

The NC analog of the usual Laplace operator is

$$
\begin{aligned}
\hat{\Delta}_{\lambda} \Psi=-\frac{1}{\lambda r}\left[\hat{a}_{\alpha}^{+},\right. & {\left.\left[\hat{a}_{\alpha}, \Psi\right]\right] } \\
& =-\frac{1}{\lambda^{2}(N+1)}\left[\hat{a}_{\alpha}^{+},\left[\hat{a}_{\alpha}, \Psi\right]\right] .
\end{aligned}
$$


As to the operator $\hat{U}$, the NC analog of the central potential, it is defined simply as the multiplication of the NC wave function by $U(r)$ :

$$
(\hat{U} \Psi)(r)=U(r) \Psi=\Psi U(r) .
$$

Since any term of $\Psi \in \mathcal{H}_{\lambda}$ consists of the same number of creation and annihilation operators (any commutator of such a term with $r$ is zero), there is no difference between left and right multiplication by $U(r)$.

So finally here is the definition of our NC Hamiltonian:

$$
\hat{H} \Psi=\frac{\hbar^{2}}{2 m \lambda r}\left[\hat{a}_{\alpha}^{+},\left[\hat{a}_{\alpha}, \Psi\right]\right]-\frac{q}{r} \Psi .
$$

The coordinate operator $\hat{x}_{j}$ acts on $\Psi$ symmetrically as

$$
\hat{x}_{j} \Psi=\frac{1}{2}\left(x_{j} \Psi+\Psi x_{j}\right) .
$$

As to the velocity operator, clearly it should be in some relation with the evolution of the coordinate operator. The NC analog of the time derivative is proportional to the commutator of the quantity considered with $H$; so the components of the velocity operator are given by

$$
\hat{V}_{j} \Psi=-i\left[\hat{x}_{j}, \hat{H}\right] \Psi .
$$

Both sets of NC observables, $\hat{V}_{j}$ and $\hat{x}_{j}$, have been introduced in [10]. As we see below, they are well adapted to the construction of the NC analog of the LRL vector.

Based on what has been briefly summarized above, the NC analog of the Schrödinger equation with the Coulomb potential in $\mathbf{R}_{\lambda}^{3}$ can be postulated:

$$
\frac{\hbar^{2}}{2 m \lambda r}\left[\hat{a}_{\alpha}^{+},\left[\hat{a}_{\alpha}, \Psi\right]\right]-\frac{q}{r} \Psi=E \Psi .
$$

To avoid overloading the formulas, we usually set $m=1, \hbar=1$ below.

\section{DyNAMiCAL SYMMETRY IN NCQM}

It is time to address the NCQM version of the Coulomb-Kepler problem. Our task is to find sensible analogs of the three components $A_{i}$ of the LRL vector in such a way that all the requirements regarding commutation relations are met (the commutator with the Hamiltonian has to be zero because of the conservation law and relations among all components of $\vec{A}$ and $\vec{L}$ are supposed to correspond to the relevant symmetry). Recall the subtlety which had to be taken into account when the standard QM version of the LRL vector had been built based on the classical model. The cross product of velocity and angular momentum needed symmetrization due to their nonvanishing commutator. The $\mathrm{NC}$ operators that we are going to use when constructing the analog of the cross product part, i.e. $\hat{V}_{i}, \hat{L}_{i}$, do not commute either, so some adjustment of this sort will also have to be made.
However, there is also another, "potential" part of the LRL vector, which is proportional to $\vec{r} / r$. The corresponding NC analogs of $x_{i}$ and $\Psi$ do not commute either, so we resolve the ordering in a similar way as in the cross product case - we take $\hat{x}_{k}$ instead of $x_{k}$ :

$$
\hat{A}_{k}=\frac{1}{2} \varepsilon_{i j k}\left(\hat{L}_{i} \hat{V}_{j}+\hat{V}_{j} \hat{L}_{i}\right)+q \frac{\hat{x}_{k}}{r} .
$$

It has turned out that besides coping with the ordering dilemma, nothing more needs to be done - that is, except for doing the calculations to show that our definition of $\hat{A}_{k}$ has been a good choice.

So now we are going to take the NC analogs of the Hamiltonian, velocity, angular momentum and position operators, and build the NC LRL vector according to 21 .

Then we have to move to the next task - evaluate the commutator $\left[\hat{A}_{i}, \hat{H}\right]$, examine the commutation relations between $\hat{A}_{k}$ and $\hat{L}_{k}$, searching for the signs of a higher dynamical symmetry. As soon as the symmetry group is recognized, we can construct the corresponding Casimir operators.

All these crucial operators: the Hamiltonian, velocity, angular momentum and position operators, have been defined already in terms of creation and annihilation operators $a_{\alpha}^{+}, a_{\alpha}$; knowing the commutation relations for these, one can calculate all that is required. However, after writing it all down and trying to make heads and tails of it, we soon realize that the problem is not assigned in the most friendly way. This definitely seems to be a case in which introducing some auxiliary quantities may help. There are certain combinations of $a_{\alpha}^{+}, a_{\alpha}$ that occur often in our expressions, and separating them the right way makes the calculations more manageable.

\subsection{Auxiliary OPERATORS}

We have to examine how the operators considered here act on the wave function $\Psi$. They are expressed in terms of $\hat{a}_{\alpha}^{+}, \hat{a}_{\alpha}$. In general it makes a difference whether the creation and annihilation operators act from the right or the left and the following notation seems to be useful to keep track of it:

$$
\begin{array}{rlrl}
\hat{a}_{\alpha} \Psi & =a_{\alpha} \Psi, & \hat{b}_{\alpha} \Psi=\Psi a_{\alpha}, \\
\hat{a}_{\alpha}^{+} \Psi=a_{\alpha}^{+} \Psi, & \hat{b}_{\alpha}^{+} \Psi=\Psi a_{\alpha}^{+} .
\end{array}
$$

An advantage of this notation is the fact that now we do not have to drag $\Psi$ into the formulas just to make clear which side the operators act from. The relevant commutation relations are (see (5))

$$
\left[\hat{a}_{\alpha}, \hat{a}_{\beta}^{+}\right]=\delta_{\alpha \beta}, \quad\left[\hat{b}_{\alpha}, \hat{b}_{\beta}^{+}\right]=-\delta_{\alpha \beta} .
$$

The other commutators are zero. This, when kept in mind, spares a lot of paper during the calculations. 
As it was already mentioned, we use the position operator in the form

$$
\begin{aligned}
\hat{x}_{i} \Psi & =\frac{1}{2}\left(x_{i} \Psi+\Psi x_{i}\right)=\frac{\lambda}{2} \sigma_{\alpha \beta}^{i}\left(\hat{a}_{\alpha}^{+} \hat{a}_{\beta}+\hat{b}_{\beta} \hat{b}_{\alpha}^{+}\right) \Psi, \\
\hat{r} \Psi & =\frac{1}{2}(r \Psi+\Psi r)=\frac{\lambda}{2}\left(\left(\hat{a}_{\alpha}^{+} \hat{a}_{\alpha}+1\right)+\left(\hat{b}_{\alpha} \hat{b}_{\alpha}^{+}+1\right)\right) \Psi .
\end{aligned}
$$

The following sequences of operators appear often and their role is important enough to admit that they deserve some notation on their own:

$$
\begin{aligned}
\hat{W}_{k} & =\sigma_{\alpha \beta}^{k}\left(\hat{a}_{\alpha}^{+} \hat{a}_{\beta}-\hat{a}_{\alpha}^{+} \hat{b}_{\beta}-\hat{a}_{\beta} \hat{b}_{\alpha}^{+}+\hat{b}_{\alpha}^{+} \hat{b}_{\beta}\right), \\
\hat{W} & =\hat{a}_{\alpha}^{+} \hat{a}_{\alpha}-\hat{a}_{\alpha}^{+} \hat{b}_{\alpha}-\hat{b}_{\alpha}^{+} \hat{a}_{\alpha}+\hat{b}_{\alpha}^{+} \hat{b}_{\alpha} \\
\hat{W}_{k}^{\prime} & =\hat{W}_{k}-2 \lambda E \hat{x}_{k} \\
\hat{W}^{\prime} & =\hat{W}-2 \lambda E \hat{r}
\end{aligned}
$$

where $E$ is energy and $\lambda$ is the NC parameter already mentioned. Note that the only difference between $\hat{W}_{k}^{\prime}$ and $\hat{W}_{k}$ is the constant multiplying one of their terms. $\hat{W}^{\prime}$ and $\hat{W}$ are related similarly.

\subsection{NC OPERATORS REVISITED}

Now we rewrite the Hamiltonian, the velocity operator and the NC LRL vector in terms of the new auxiliary operators which have been introduced.

$$
\begin{gathered}
\hat{H}=\frac{1}{2 \lambda \hat{r}}\left(\hat{a}_{\alpha}^{+} \hat{a}_{\alpha}+\hat{b}_{\alpha}^{+} \hat{b}_{\alpha}-\hat{a}_{\alpha}^{+} \hat{b}_{\alpha}-\hat{a}_{\alpha} \hat{b}_{\alpha}^{+}\right)-\frac{q}{\hat{r}} \\
=\frac{1}{2 \lambda \hat{r}} \hat{W}-\frac{q}{\hat{r}}, \\
\hat{V}_{i}=-i\left[\hat{x}_{i}, \hat{H}\right]=\frac{i}{2 \hat{r}} \sigma_{\alpha \beta}^{i}\left(\hat{a}_{\alpha}^{+} \hat{b}_{\beta}-\hat{a}_{\beta} \hat{b}_{\alpha}^{+}\right) \\
\hat{A}_{k}=\frac{1}{2} \varepsilon_{i j k}\left(\hat{L}_{i} \hat{V}_{j}+\hat{V}_{j} \hat{L}_{i}\right)+q \frac{\hat{x}_{k}}{\hat{r}} \\
=\frac{1}{2 \hat{r} \lambda}\left(\hat{r} \hat{W}_{k}^{\prime}-\hat{x}_{k}\left(\hat{W}^{\prime}-2 \lambda q\right)\right) .
\end{gathered}
$$

Deriving equations (28) and (29) involves somewhat laborious calculations. The details can be found in [7.

This gives us an opportunity to write the NC Schrödinger equation in the following way:

$$
\left(\frac{1}{2 \lambda \hat{r}} \hat{W}-\frac{q}{\hat{r}}-E\right) \Psi_{E}=\frac{1}{2 \lambda \hat{r}}\left(\hat{W}^{\prime}-2 \lambda q\right) \Psi_{E}=0 .
$$

$\Psi_{E}$ belongs to $\mathcal{H}_{\lambda}^{E}$, i.e. to the subspace spanned by the eigenvectors of the Hamiltonian.

The important quantity for us is $\left.\hat{A}_{k}\right|_{\mathcal{H}^{E}}$, the LRL vector as it acts on the solutions of the Schrödinger equation:

$$
\left.\hat{A}_{k}\right|_{\mathcal{H}_{\lambda}^{E}}=\frac{1}{2 \hat{r} \lambda}(\hat{r} \hat{W}_{k}^{\prime}-\hat{x}_{k} \underbrace{\left(\hat{W}^{\prime}\right.}_{\text {see Eq. } \sqrt[30]{\left.W^{\prime}-2 \lambda q\right)}})=\frac{1}{2 \lambda} \hat{W}_{k}^{\prime} .
$$

When dealing with calculations related to the conservation of $\hat{A}_{k}$, we just need to ascertain whether the following commutator with the Hamiltonian vanishes:

$$
\begin{array}{r}
\dot{\hat{W}}_{k}^{\prime}=i\left[\hat{H}_{0}-\frac{q}{\hat{r}}, \hat{W}_{k}^{\prime}\right]=i\left[\frac{1}{2 \hat{r} \lambda} \hat{W}^{\prime}-\frac{q}{\hat{r}}, \hat{W}_{k}^{\prime}\right] \\
=\frac{i}{2 \hat{r} \lambda}\left[\hat{W}^{\prime}, \hat{W}_{k}^{\prime}\right]+i\left[\frac{1}{\hat{r}}, \hat{W}_{k}^{\prime}\right]\left(\frac{\hat{W}^{\prime}}{2 \lambda}-q\right) \\
=0 .
\end{array}
$$

The second term in the second-to-last line vanishes when acting on vectors from $\mathcal{H}_{\lambda}^{E}$ (and we are not so interested in the rest of $\mathcal{H}_{\lambda}$ ). The first term proportional to $\left[\hat{W}^{\prime}, \hat{W}_{k}^{\prime}\right]$ does not contribute either. The calculations proving this involve more steps and can be found in 7 .

The equation above encourages one to search for the underlying $S O(4)$ symmetry, since the LRL vector conservation makes its components suitable candidates for half of its generators, the remaining three consisting of the components of the angular momentum. Once again we have to ask the reader either to check [7] for details or simply to believe that the following holds:

$$
\left[\hat{A}_{i}, \hat{A}_{j}\right]=i \varepsilon_{i j k}\left(-2 E+\lambda^{2} E^{2}\right) \hat{L}_{k}
$$

There is nothing but a constant in the way, as long as we let the operator $\left[\hat{A}_{i}, \hat{A}_{j}\right]$ act upon the vectors from $\mathcal{H}_{\lambda}^{E}$ with the energy fixed. Eq. 33 and

$$
\left[\hat{L}_{i}, \hat{L}_{j}\right]=i \varepsilon_{i j k} \hat{L}_{k}, \quad\left[\hat{L}_{i}, \hat{A}_{j}\right]=i \varepsilon_{i j k} \hat{A}_{k}
$$

define Lie algebra relations corresponding to a particular symmetry group, the actual form of which depends on the sign of the $E$-dependent factor in $(33)$. The relevant relations for $\hat{L}_{i}$ have already been mentioned, the formula for the mixed commutator $\left[\hat{L}_{i}, \hat{A}_{j}\right]$ follows from the fact that $\hat{A}_{j}, j=1,2,3$, are components of a vector.

There are three independent cases:

- $S O(4)$ symmetry:

$$
-2 E+\lambda^{2} E^{2}>0 \Longleftrightarrow E<0 \text { or } E>2 / \lambda^{2} ;
$$

- $S O(3,1)$ symmetry:

$$
-2 E+\lambda^{2} E^{2}<0 \Longleftrightarrow 0<E<2 / \lambda^{2} ;
$$

- $E(3)$ Euclidean group:

$$
-2 E+\lambda^{2} E^{2}=0 \Longleftrightarrow E=0 \text { or } E=2 / \lambda^{2} .
$$

The admissible values of $E$ should correspond to the unitary representations of the symmetry in question. This requirement guarantees that the generators $\hat{L}_{j}$ and $\hat{A}_{j}$ are realized as hermitian operators, and consequently correspond to physical observables. The Casimir operators in the mentioned cases are

$$
\begin{aligned}
& \hat{C}_{1}^{\prime}=\hat{L}_{j} \hat{A}_{j}, \\
& \hat{C}_{2}^{\prime}=\hat{A}_{i} \hat{A}_{i}+\left(-2 E+\lambda^{2} E^{2}\right)\left(\hat{L}_{i} \hat{L}_{i}+1\right) .
\end{aligned}
$$


The prime indicates that we are not using the standard normalization of Casimir operators.

Now, we need to calculate their values in $\mathcal{H}_{\lambda}^{E}$. The first Casimir vanishes in all cases due to the fact that $\hat{C}_{1}^{\prime} \Psi_{E} \sim r \Psi_{E}-\Psi_{E} r=0$. The second Casimir operator is somewhat more demanding. According to (31) we have

$$
\begin{aligned}
\hat{C}_{2}^{\prime} \Psi_{E}=\left(\frac{\hat{W}_{i}^{\prime} \hat{W}_{i}^{\prime}}{4 \lambda^{2}}+(-2 E+\right. & \left.\left.\lambda^{2} E^{2}\right)\left(\hat{L}_{i} \hat{L}_{i}+1\right)\right) \Psi_{E} \\
& =\frac{1}{4 \lambda^{2}} \hat{W}^{\prime} \hat{W}^{\prime} \Psi_{E},
\end{aligned}
$$

where we used the quadratic identity

$$
\hat{W}_{i}^{\prime} \hat{W}_{i}^{\prime}+4 \lambda^{2}\left(-2 E+\lambda^{2} E^{2}\right)\left(\hat{L}_{i} \hat{L}_{i}+1\right)=\hat{W}^{\prime 2} .
$$

According to the Schrödinger equation, $\left(\hat{W}^{\prime}\right)^{2} \Psi_{E}=$ $4 \lambda^{2} q^{2} \Psi_{E}$, and we are left with

$$
\hat{C}_{2}^{\prime} \Psi_{E}=q^{2} \Psi_{E}
$$

Since both Casimir operators take constant values $\hat{C}_{1}^{\prime}=0$ and $\hat{C}_{2}^{\prime}=q^{2}$ in $\mathcal{H}_{\lambda}^{E}$, we are dealing with irreducible representations of the dynamical symmetry group $G$ that are unitary for particular values of energy. In all the cases considered, $G=S O(4), S O(3,1), E(3)$, the unitary irreducible representations are well known. The corresponding systems of eigenfunctions that span the representation space have been found in [9]. Here we do not repeat their construction, but we restrict ourselves to brief comments pointing out some interesting aspects.

\subsection{Bound STATES - THE CASE OF $S O(4)$ SYMMETRY $-2 E+\lambda^{2} E^{2}>0$}

In this case we rescale the LRL vector as

$$
\hat{K}_{j}=\frac{\hat{A}_{j}}{\sqrt{-2 E+\lambda^{2} E^{2}}}=\frac{\hat{W}_{j}^{\prime}}{2 \lambda \sqrt{-2 E+\lambda^{2} E^{2}}} .
$$

After this step Eqs. 33, 34 turn into the following relations:

$$
\begin{aligned}
{\left[\hat{L}_{i}, \hat{L}_{j}\right] } & =i \varepsilon_{i j k} \hat{L}_{k}, \\
{\left[\hat{L}_{i}, \hat{K}_{j}\right] } & =i \varepsilon_{i j k} \hat{K}_{k}, \\
{\left[\hat{K}_{i}, \hat{K}_{j}\right] } & =i \varepsilon_{i j k} \hat{L}_{k} .
\end{aligned}
$$

Thus we have got the representation of the so(4) algebra. The relevant normalized Casimir operators are

$$
\hat{C}_{1}=\hat{L}_{j} \hat{K}_{j}, \quad \hat{C}_{2}=\hat{K}_{i} \hat{K}_{i}+\hat{L}_{i} \hat{L}_{i}+1
$$

As we have stated already, the $\hat{C}_{1}$ acting on an eigenfunction of the Hamiltonian returns zero. As to $\hat{C}_{2}$, we know that for $s o(4)$, under the condition that the first Casimir is zero, the second Casimir has to be equal to $n^{2}$ for some integer $n=j+1, j+2, \ldots$ (with $j(j+1)$ corresponding to the square of the angular momentum). At the same time, according to (38) it is related to the energy:

$$
\hat{K}_{i} \hat{K}_{i}+\hat{L}_{i} \hat{L}_{i}+1=\frac{q^{2}}{\lambda^{2} E^{2}-2 E}=n^{2} .
$$

Now solving the quadratic equation for energy we obtain two discrete sets of solutions depending on $n$ :

$$
E=\frac{1}{\lambda^{2}} \mp \frac{1}{\lambda^{2}} \sqrt{1+\kappa_{n}^{2}}, \quad \kappa_{n}=\frac{q \lambda}{n} .
$$

The first set of eigenfunctions of the Hamiltonian in (17) for energies $E<0$ (i.e. negative sign in front of the square root in (43) ) has been found for Coulomb attractive potential, i.e. $q>0$ in $(17)$ :

$$
E_{\lambda n}^{I}=\frac{1}{\lambda^{2}}-\frac{1}{\lambda^{2}} \sqrt{1+\kappa_{n}^{2}} .
$$

These eigenvalues possess a smooth standard limit for $\lambda \rightarrow 0$

$$
\begin{array}{r}
E_{\lambda n}^{I}=\frac{1}{\lambda^{2}}-\frac{1}{\lambda^{2}}\left(1+\frac{1}{2} \kappa_{n}^{2}-\frac{1}{24} \kappa_{n}^{4}+\cdots\right) \\
\rightarrow-\frac{q^{2}}{2 n^{2}}=-\frac{q^{2} m}{2 n^{2} \hbar^{2}} .
\end{array}
$$

This spectrum coincides (in the commutative limit $\lambda \rightarrow 0$ ) with the spectrum for Coulomb attractive potential, $q>0$, that was found by Pauli using algebraic methods prior to solving Schrödinger equation for the hydrogen atom.

The full set of eigenfunctions of (17) for energies $E<0$ was constructed in 9 by explicitly solving the NC Schrödinger equation. The radial NC wave functions defined in (14) are given in terms of the hypergeometric function

$$
\begin{aligned}
R_{\lambda n}^{I} & =\left(\Omega_{n}\right)^{N} F\left(-n,-N, 2 j+2,-2 \kappa_{n} \Omega_{n}^{-1}\right), \\
\Omega_{n} & =\frac{\kappa_{n}-\sqrt{1+\kappa_{n}^{2}}+1}{\kappa_{n}+\sqrt{1+\kappa_{n}^{2}}-1},
\end{aligned}
$$

where $N=a_{\alpha}^{+} a_{\alpha}$ controls the radial NC variable.

The second set of very unexpected solutions corresponds to energies (43) with positive sign

$$
E_{\lambda n}^{I I}=\frac{1}{\lambda^{2}}+\frac{1}{\lambda^{2}} \sqrt{1+\kappa_{n}^{2}}>\frac{2}{\lambda^{2}} .
$$

The corresponding radial $\mathrm{NC}$ wave functions have been found in 9] solving NC Schrödinger equation for a Coulomb repulsive potential, $q<0$ in (17). These radial $\mathrm{NC}$ wave functions are closely related to those given above

$$
R_{\lambda n}^{I I}=\left(-\Omega_{n}\right)^{N} F\left(-n,-N, 2 j+2,2 \kappa_{n} \Omega_{n}^{-1}\right) .
$$

Both $S O(4)$ representations, the representation for Coulomb attractive potential with $E_{\lambda n}^{I}<0$ and that for ultra-high energies $E_{\lambda n}^{I I}>2 / \lambda^{2}$ for Coulomb repulsive potential, are unitary equivalent as in both representations the Casimir operators take the same values, $\hat{C}_{1}=0$ and $\hat{C}_{1}=n^{2}$. However, physically they are quite distinct: In the commutative limit $\lambda \rightarrow 0$ the first bound states persist and reduce to the standard ones, while the extraordinary bound states at ultra-high energies disappear from the Hilbert space. 


\subsection{Coulomb ScAttering - The CASe $2 E-\lambda^{2} E^{2}>0$}

In this case we rescale the LRL vector as

$$
\hat{K}_{j}=\frac{\hat{A}_{j}}{\sqrt{2 E-\lambda^{2} E^{2}}}=\frac{\hat{W}_{j}^{\prime}}{2 \lambda \sqrt{2 E-\lambda^{2} E^{2}}} .
$$

After this step we obtain equations

$$
\begin{aligned}
{\left[\hat{L}_{i}, \hat{L}_{j}\right] } & =i \varepsilon_{i j k} \hat{L}_{k}, \\
{\left[\hat{L}_{i}, \hat{K}_{j}\right] } & =i \varepsilon_{i j k} \hat{K}_{k}, \\
{\left[\hat{K}_{i}, \hat{K}_{j}\right] } & =-i \varepsilon_{i j k} \hat{L}_{k} .
\end{aligned}
$$

So this time we have obtained the representation of the $s o(3,1)$ algebra. The relevant normalized Casimir operators are

$$
\hat{C}_{1}=\hat{L}_{j} \hat{K}_{j}, \quad \hat{C}_{2}=\hat{K}_{i} \hat{K}_{i}-\hat{L}_{i} \hat{L}_{i} .
$$

In our case $\hat{C}_{1}=0$, so we are dealing with $S O(3,1)$ unitary representations that are labeled by the value of second Casimir operator $\hat{C}_{2}$. Rewriting (38) in terms of $\hat{K}_{j}$ we obtain relation between energy $E$ and the eigenvalue $\tau$ of $\hat{C}_{2}$ :

$$
\hat{K}_{i} \hat{K}_{i}-\hat{L}_{i} \hat{L}_{i}=1+\frac{q^{2}}{2 E-\lambda^{2} E^{2}}=\tau>1 .
$$

Thus we are dealing with the spherical principal series of $S O(3,1)$ unitary representations, see e.g [11].

The scattering NC wave functions have been constructed in [9] for any admissible energy $E \in\left(0,2 / \lambda^{2}\right)$, and from their asymptotic behavior the partial wave $S$-matrix has been derived

$$
S_{j}^{\lambda}(E)=\frac{\Gamma\left(j+1-i \frac{q}{p}\right)}{\Gamma\left(j+1+i \frac{q}{p}\right)}, \quad p=\sqrt{2 E-\lambda^{2} E^{2}} .
$$

It can be easily seen that such $S$-matrix possesses poles at energies $E=E_{\lambda n}^{I}$ for Coulomb attractive potential and poles at energies $E=E_{\lambda n}^{I I}$ for Coulomb repulsive potential, where both $E_{\lambda n}^{I}$ and $E_{\lambda n}^{I I}$ coincide with (44) and (47) given above. As for energies

$$
E_{\mp}^{\lambda}=\frac{1}{\lambda^{2}}\left(1 \mp \sqrt{1-\frac{\lambda^{2} q^{2}}{\tau-1}}\right)
$$

the Casimir operator values coincide, the corresponding representations are unitarily equivalent. This relates the scattering for low energies $0<E<1 / \lambda^{2}$ to that at high energies $1 / \lambda^{2}<E<2 / \lambda^{2}$.

We skip the limiting cases of the scattering at the edges $E=0$ and $E=2 / \lambda^{2}$ of the admissible interval of energies, where the $S O(3,1)$ group contracts to the Euclidean group $E(3)=S O(3) \triangleright T(3)$ of isometries of $3 \mathrm{D}$ space. The corresponding NC Hamiltonian eigenstates are given in [9].

\section{Conclusions}

This paper deals with the Coulomb-Kepler problem in noncommutative space. We have found the NC analog of the LRL vector; its components, together with those of the NC angular momentum operator, supply the algebra of generators of a symmetry group. It is interesting that the formula for the $\mathrm{NC}$ version of the LRL vector looks very much like the one from standard QM, that is, when written in terms of the proper NC observables: NC angular momentum, NC velocity, symmetrized $\mathrm{NC}$ coordinate and $\mathrm{NC}$ radial distance.

It is quite remarkable that the $S O(4)$ symmetry has appeared twice: Firstly, not so surprisingly, when addressing the problem of the bound states for negative energies in the case of the attractive Coulomb potential. These have an analog in standard quantum mechanics, and our result for negative energy bound states indeed coincides with the well known QM prediction if the commutative limit $\lambda \rightarrow 0$ is applied. The second appearance of the $S O(4)$ symmetry is probably not so expected, for we have found a set of bound states for positive energies above a certain ultra-high value in the case that the potential is repulsive. However, there is again no discrepancy between QM and NCQM, since the unexpected ultra-high energy bound states disappear from the Hilbert space in the above mentioned commutative limit.

When examining the scattering (relevant for the interval of energies between zero and the mentioned critical ultra-high value), $S O(3,1)$ is the symmetry to be considered. The scattering is usually characterized by the $S$-matrix. In the $\mathrm{NC}$ version of the problem this object has exactly those poles in complex energy plane which correspond to the bound states (of both kinds) mentioned above. This goes, however, beyond the scope of this paper.

To summarize, there are basically two ways of examining the hydrogen energy spectrum: by solving some differential equation in Schrödinger fashion or by looking for an underlying symmetry and using an algebraic approach à la Pauli. Both possibilities were tried in NCQM (the aim of this paper has been mainly to provide some outline of the latter option), for details see 7], 9]. We are glad to find out that both approaches (agreeing in standard QM) lead to the same outcomes also in NCQM.

\section{ACKNowledgements}

The author V. G. is indebted to Comenius University for support received from grant No. UK/545/2013.

\section{REFERENCES}

[1] Jakob Hermann, Giornale de Letterati D'Italia 2 (1710) 447; Jakob Hermann, Extrait d'une lettre de M. Herman à $M$. Bernoulli datée de Padoüe le 12. Juillet 1710, Histoire de l'academie royale des sciences (Paris) 1732: 519; 
[2] Johann Bernoulli, Extrait de la Réponse de $M$. Bernoulli à M. Herman datée de Basle le \%. Octobre 1710, Histoire de l'academie royale des sciences (Paris) 1732: 521 .

[3] P. S. Laplace, Traité de mécanique céleste, Tome I (1799), Premiere Partie, Livre II, pp.165ff.

[4] C. Runge, Vektoranalysis, Volume I, Leipzig Hirzel (1919).

[5] W. Lenz, Zeitschrift für Physik 24 (1924) 197, Über den Bewegungsverlauf und Quantenzustände der gestörten Keplerbewegung.

[6] W. Pauli, Zeitschrift für Physik 36 (1926) 336, Über das Wasserstoffspektrum vom Standpunkt der neuen Quantenmechanik.

[7] Veronika Gáliková, S. Kováčik and P. Prešnajder, Laplace-Rung-Lenz vector in quantum mechanics in noncommutative space - J. Math. Phys. 54, 122106 (2013) DOI: $10.1063 / 1.4835615$
[8] F. A. Berezin, Commun. Math. Phys. 40 (1975) 153 DOI: 1007/BF01609397 J. Hoppe, Elem. Part. Res. J. 80 (1989) 145; J. Madore, Journ. Math. Phys. 32 (1991) 332 DOI: 10.1063/1.529418 H. Grosse and P. Prešnajder, Lett. Math. Phys. 28 (1993) 239 DOI: 10.1007/BF00745155; H. Grosse, C. Klimčík and P. Prešnajder, Int. J. Theor. Phys. 35 (1996) 231 DOI: 10.1007/BF02083810.

[9] Veronika Gáliková and Peter Prešnajder, Coulomb problem in non-commutative quantum mechanics J. Math. Phys. 54, 052102 (2013) DOI: 10.1063/4803457,

[10] S. Kováčik and P. Prešnajder, The velocity operator in quantum mechanics in noncommutative space, J. Math. Phys. 54, 102103 (2013) DOI: 1063/1.4826355

[11] A. O. Barut and R. Raçzka, Theory of Group Representations and Applications, Polish Scientific Publishers, 1977. 